\title{
Evaluation of F1 Hybrids along with Parents for Yield and Related Characteristics in Tomato (Solanum lycopersicum Child)
}

\author{
Sanket Kumar $^{1 *}$, Vikas Singh ${ }^{3}$, Praveen Kumar Maurya ${ }^{2}$, \\ B. Ashok Kumar ${ }^{2}$ and P.K. Yadav ${ }^{1}$ \\ ${ }^{1}$ Department of Vegetable Science, College of Horticulture and Forestry, Central agricultural \\ University, Pasighat, East Siang, 791102, Arunachal Pradesh, India \\ ${ }^{2}$ Department of Vegetable Science, Faculty of Horticulture, Bidhan Chandra Krishi \\ Viswavidyalaya, Mohanpur, Nadia, West Bengal, India \\ ${ }^{3}$ ICAR-IIVR-RRS, Sargatia, Kushinagar, U.P., India \\ *Corresponding author
}

\section{A B S T R A C T}

\begin{tabular}{|c|c|}
\hline & \multirow{8}{*}{$\begin{array}{l}\text { Eight diverse homozygous tomato parental lines were crossed in half diallel fashion, to } \\
\text { develop twenty-eight hybrids. These } 28 \mathrm{~F}_{1} \text { hybrids and their parents were evaluated at } \\
\text { Department of Vegetable Science, C.H.F., CAU, Pasighat, A.P. during } 2014-2015 \text {. A wide } \\
\text { range of variation was observed for fruit yield and related constituents. The high PCV, } \\
\text { GCV, ECV, heritability (broad sense), and genetic advance over percentage of mean were } \\
\text { recorded for fruit yield per plant. DVRT- } 1 \times \text { CHFT-50 was found earliest flowering in } \\
50.67 \text { days and earliest harvest in } 70.00 \text { days. Earliest flowering concurrent with early fruit } \\
\text { set that are expressed in all hybrids under studied. The highest fruit yield was established } \\
\text { in DVRT- } 2 \times \text { CHFT- } 77(9.13 \mathrm{~kg}) \text {, followed by DVRT- } 2 \times \mathrm{H}-86(6.86 \mathrm{~kg}) \text { and CHFT- } 60 \times \\
\text { CHFT-71 }(5.92 \mathrm{~kg}) \text {. Among the hybrids, the fruit weight was correlated with fruit yield. } \\
\text { Cross, H- } 86 \times \text { CHFT-50 was given high yield along with quality characters. The hybrid } \\
\text { DVRT- } 2 \times \text { CHFT-77, H- } 86 \times \text { CHFT- } 50 \text { and CHFT- } 60 \times \text { CHFT- } 71 \text { were found best } \\
\text { regarding fruit yield, quality traits and earliness. Therefore, the current study showed that } \\
\text { hybrid breeding approaches strengthening to improve yield, earliness together with } \\
\text { qualities. }\end{array}$} \\
\hline & \\
\hline & \\
\hline Range, PCV, GCV. & \\
\hline Article Info & \\
\hline $\begin{array}{l}\text { Accepted: } \\
26 \text { August } 2017\end{array}$ & \\
\hline $\begin{array}{l}\text { Available Online: } \\
10 \text { September } 2017\end{array}$ & \\
\hline & \\
\hline
\end{tabular}

\section{Introduction}

Now a day, population and demand both are escalating day by day. The major challenges in agriculture research are the ways and means of achieving food and nutritional security (Swaminathan and Bhavani, 2013). Production of enough quantity and quality of vegetables holds the key to overcome the situation. Tomato (Solanum lycopersicum Child, $2 n=2 x=24$ ) is one of the most popular and commercially cultivated annual herbaceous vegetable, grown in tropical and subtropical regions worldwide (Rao et al., 2007). It is estimated that the total tomato area and production in India were 0.76 million hectares and 18.39 million tonnes, respectively (Anonymous, 2015). It is rich in medicinal value having antiseptic, antioxidant and anticancerous properties. Tomato being predominantly bisexual self-pollinated crop, does not suffer much from inbreeding 
depression and has the advantage of producing a large number of seed per fruit, facilitating hybrid breeding through reasonably low cost of hybrid seed production. For proper evaluation of genetic resources is essential to understand the genetic variability and heritability. Due to increasing popularity of $F_{1}$ hybrids in tomato, it is need to obtain such hybrids that have excellent qualities and yield together with resistance to biotic and abiotic stresses. The $\mathrm{F}_{1}$ hybrids and their parents for yield related traits were also evaluated by Chattopadhyay et al., (2013); Jindal et al., (2015); Panthee et al., (2015); Lekshmi and Celine (2015) and Pandey et al., (2016). The major objectives are (1) to study the genetic variability, heritability and genetic advance for yield and yield components and (2) to develop parents and hybrids having high yield along with earliness and quality traits.

\section{Materials and Methods}

The experiment was conducted at Vegetable Experimental Farm, College of Horticulture and Forestry, Central Agricultural University, Pasighat, East Siang, Arunachal Pradesh, India. The experimental materials for the present study comprised of eight elite diverse and homozygous pure lines/varieties of tomato parental lines namely DVRT-1, DVRT-2, H-86, CHFT-50, CHFT-60, CHFT71, CHFT-77 and CHFT-79 were crossed in all possible combinations in half diallel technique excluding reciprocals [n(n-1)/2] during Rabi, 2013-2014. The parents and hybrids were evaluated in the successive year (2014-15) for sixteen quantity and quality traits i.e., days to first flowering (DFF), days to $50 \%$ flowering (DF), days to $50 \%$ fruit set (DFS), days to first harvest (DFH), number of primary branches per plant (NPB), number of locules per fruit (NLF), pericarp thickness (PT), plant height $(\mathrm{PH})$, fruit length (FL), fruit girth (FG), fruit weight (FW), number of fruits per plant (NFP), fruit yield per plant (FYP), total soluble solids (TSS), lycopene content (LC) and $\beta$-carotene content (BC). Observations were recorded on five randomly selected plants from each hybrid in each replication. The recorded observations were averaged to get mean values. Analysis of variance for Randomized Block Design was carried out according to following procedure as suggested by Gomez and Gomez (1983). The genotypic and phenotypic coefficients of variation were estimated by Burton (1952). Heritability in broad sense was computed according to Allard (1960). Genetic advance and genetic advance as per cent of mean was calculated using the method of Johnson et al., (1955).

\section{Results and Discussion}

The mean square differences due to parents vs. hybrids were found to be significant for seven characters (Table 1). Analysis of variance due to treatments and hybrids revealed significant differences for all the characters, suggested great variability in the source of variations for almost all the characters under studied. These results are in accordance with Pedapati et al., (2014) except for yield and some yield related traits. Mean performance of 8 parental lines and $28 \mathrm{~F}_{1}$ hybrids for all the traits (Tables 2, 3 and 4) revealed a wide range of mean values, which indicated that the parental lines involved in this study were genetically diverse and had good breeding value, which confirmed the predictions of analysis of variance.

The estimates of genotypic coefficient of variation (Table 1) was the highest for $\beta$ carotene (46.25), followed by lycopene content (45.37) and fruit yield per plant (44.82), whereas the lowest GCV was found for days to first harvest (4.64), followed days to first flowering (6.91) and days to $50 \%$ fruit set (7.19). The results were in concurrence 
with Ahmad et al., (2016). The highest phenotypic coefficient of variation was recorded for fruit yield per plant (47.13), followed by $\beta$-carotene (46.51), and lycopene content (45.59), whereas the lowest PCV was recorded for days to first harvest (8.40), T.S.S. (8.96) and days to $50 \%$ fruit set (10.78). The values of PCV were higher than GCV obtained for all traits under studied (Table 1). Prajapati et al., (2015) also reported the higher PCV value than GCV for most of the characters. The highest and lowest ECV were found for fruit yield per plant (14.57) and lycopene content (4.52), respectively. Genotypic coefficient of variation shows the extent of genetic variability in a population, was high for all the characters.

The highest heritability and genetic advance over percentage of mean were recorded on lycopene content (99.02 and 93.01\%), $\beta$ carotene $(98.88$ and $94.74 \%)$ and fruit yield per plant ( 90.45 and $87.82 \%$ ). However, the lowest heritability was that of days to first harvest $(30.51 \%)$ with an expected genetic advance over percentage of mean of $5.28 \%$ (Table 1). High heritability also reported by Meeena and Bahadur (2014) and Yadav et al., (2016). Most of the characters have high heritability estimates that they will be affected by environmental condition.

The range for days to first flowering was from 46.00 to 55.33 days with a mean value of 50.46 days in parents. In case of hybrids, average mean value was 50.46 days through a range of 43.67 to 68.00 days. The results were in confirmation with Singh et al., (2014). Parent DVRT-1 showed earliest 50\% flowering and fruit set with 53.00 and 56.67 days, respectively. The best $F_{1}$ hybrid for early flowering and fruit set was DVRT-1 $\mathrm{x}$ CHFT-50 (50.67 and 54.67 days), which was followed by CHFT-77 $\times \mathrm{H}-86$ (51.33 and 55.33 days) and CHFT-60 $\times$ CHFT-71 (52.33 and 56.67 days). Similar results were also obtained by Islaam et al., (2014) and Lekshmi and Celine (2015). The promising $\mathrm{F}_{1}$ hybrid for early harvest was DVRT-1 x CHFT-50 (70.00 days), which was parity with CHFT-77 $\times$ CHFT-71 (71.67 days) and CHFT-77 $\times$ H86 (72.67 days). These kinds of results are also reported by Chernet et al., (2014).

Among the parents, the primary branches per plant varied from 7.07 to 9.33 with a mean value of 8.18 . However, for the hybrids, it was in the range of 8.20 to 13.33 with a mean value of 9.58. Parent CHF-60 recorded maximum number of primary branches per plant (9.33), which was statistically at par with DVRT-2 (8.80), CHFT-77 (8.67), DVRT-1(8.33), H-86 (8.13) and CHFT-79 (7.87). The above results are in accordance with Shankar et al., (2013) and Yadav et al., (2013). The maximum number of locules per fruit was recorded for DVRT-2 x CHFT-77 (6.33) followed by DVRT-1 x CHFT-77 (4.73) and CHFT-79 x H-86 (4.53). High magnitude of number of locules per fruit has been reported by Pandey et al., (2016). Among the parental lines, maximum pericarp thickness recorded for CHFT-79 $(0.51 \mathrm{~cm})$. However, thickest pericarp was recorded for hybrids DVRT-2 x H-86 $(0.70 \mathrm{~cm})$, which was statistically at par with DVRT-2 $x$ CHFT$77(0.68 \mathrm{~cm})$, CHFT-79 x CHFT-71 $(0.67 \mathrm{~cm})$ and CHFT-77 $\mathrm{x}$ CHFT-79 (0.63 cm). Chattopadhyay et al., (2013) and Jindal et al., (2015) also reported the similar findings. Out of 28 hybrids, maximum plant height was recorded for CHFT-60 x CHFT-50 (119.87 $\mathrm{cm})$ followed by CHFT-60 $\times$ CHFT-71 $(117.45 \mathrm{~cm})$, DVRT-2 $\times$ CHFT-79 (112.20 $\mathrm{cm})$, DVRT-2 $\times$ CHFT-60 $(108.93 \mathrm{~cm})$ and H-86 $\times$ CHFT-50 (108.12 cm). Similar observation was also made by Farooq et al., (2012) and Jindal et al., (2015). Longest fruits were recorded in the parent CHFT-50 (5.16 $\mathrm{cm})$. In the hybrids, DVRT-2 x CHFT-77 $(5.55 \mathrm{~cm})$ showed maximum fruit length fruit girth and fruit weight. 
Table.1 Analysis of variance (mean squares), PCV, GCV, ECV, $\mathrm{h}^{2}$ (broad sense), GA and GA as $\%$ of mean for sixteen characters of tomato

\begin{tabular}{|c|c|c|c|c|c|c|c|c|c|c|c|c|}
\hline Characters & Replications & Treatments & Parents & Hybrids & $\begin{array}{c}\text { Parent vs. } \\
\text { Hybrids }\end{array}$ & Error & PCV & GCV & ECV & $\begin{array}{c}\mathbf{h}^{2} \\
\text { (broad sense) }\end{array}$ & GA & $\begin{array}{l}\text { GA as \% } \\
\text { of mean }\end{array}$ \\
\hline DFF & 0.36 & $64.01 * *$ & 29.71 & $75.00 * *$ & 7.29 & 26.78 & 12.28 & 6.91 & 10.16 & 31.67 & 4.08 & 8.02 \\
\hline DF & 44.18 & $81.14 * *$ & $98.80 * *$ & $79.34 * *$ & 5.97 & 26.62 & 11.62 & 7.40 & 8.96 & 40.57 & 5.59 & 9.71 \\
\hline DFS & 10.12 & $84.72 * *$ & $102.95 * *$ & $82.75 * *$ & 10.17 & 24.91 & 10.78 & 7.19 & 8.04 & 44.45 & 6.13 & 9.87 \\
\hline DFH & 8.79 & $70.33 * *$ & 25.43 & $84.48 * *$ & 2.54 & 30.35 & 8.40 & 4.64 & 7.01 & 0.31 & 4.15 & 5.28 \\
\hline NPB & 0.47 & $5.60 * *$ & 1.78 & $5.45 * *$ & $36.46 * *$ & 1.11 & 17.42 & 13.20 & 11.37 & 57.4 & 1.91 & 20.60 \\
\hline NLF & 0.05 & $1.35 * *$ & $1.97 * *$ & $1.24 * *$ & 0.01 & 0.15 & 19.34 & 16.42 & 10.23 & 72.04 & 1.10 & 28.71 \\
\hline PT & 0.00 & $0.02 * *$ & 0.00 & $0.02 * *$ & $0.15 * *$ & 0.00 & 16.03 & 12.96 & 9.42 & 65.42 & 0.11 & 21.59 \\
\hline PH & 152.90 & $948.66 * *$ & $494.76 * *$ & $765.56 * *$ & $9069.71 * *$ & 78.94 & 22.75 & 20.17 & 10.52 & 78.6 & 31.09 & 36.84 \\
\hline FL & 0.35 & $0.56^{* *}$ & $0.84 * *$ & $0.51 * *$ & 0.07 & 0.22 & 12.89 & 7.54 & 10.45 & 34.25 & 0.41 & 9.10 \\
\hline FG & 0.40 & $0.97 * *$ & $0.63 * *$ & $1.07 * *$ & 0.42 & 0.27 & 12.65 & 8.60 & 9.27 & 46.24 & 0.67 & 12.06 \\
\hline FW & 60.24 & $2612.57 * *$ & $961.33 * *$ & $3117.01 * *$ & 551.62 & 149.71 & 35.32 & 32.48 & 13.87 & 84.58 & 54.28 & 61.53 \\
\hline NFP & 139.95 & $515.31 * *$ & 16.60 & $578.25 * *$ & $2307.03 * *$ & 59.63 & 27.25 & 23.09 & 14.46 & 71.8 & 21.51 & 40.31 \\
\hline FYP & 0.42 & $8.97 * *$ & $1.90 * *$ & $10.27 * *$ & $23.62 * *$ & 0.31 & 47.13 & 44.82 & 14.57 & 90.45 & 3.33 & 87.82 \\
\hline TSS & 0.01 & $0.26 * *$ & $0.10^{*}$ & $0.31 * *$ & 0.00 & 0.04 & 8.96 & 7.33 & 5.15 & 66.94 & 0.46 & 12.36 \\
\hline $\mathbf{L C}$ & 0.16 & $42.54 * *$ & $19.54 * *$ & $49.60 * *$ & $12.99 * *$ & 0.14 & 45.59 & 45.37 & 4.52 & 99.02 & 7.71 & 93.01 \\
\hline BC & 0.00 & $2.15^{* *}$ & $2.21 * *$ & $2.06 * *$ & $4.30 * *$ & 0.01 & 46.51 & 46.25 & 4.91 & 98.88 & 1.73 & 94.74 \\
\hline
\end{tabular}

*, ** Significant at 5 and 1 per cent probability level, respectively. PCV- Phenotypic Coefficient of Variation, GCV- Genotypic Coefficient of Variation, ECVEnvironmental Coefficient of Variation, $\mathrm{h}^{2}$ - Heritability, GA- Genetic Advance (5\%) 
Table.2 Range, mean values, top 3 parents and 5 best hybrids for yield and its attributing traits

\begin{tabular}{|c|c|c|c|c|c|c|}
\hline \multicolumn{2}{|c|}{ Particulars } & $\begin{array}{c}\text { Days to first } \\
\text { flowering }\end{array}$ & $\begin{array}{c}\text { Days to 50\% } \\
\text { flowering }\end{array}$ & Days to $50 \%$ fruit set & Days to first harvest & $\begin{array}{l}\text { Number of primary } \\
\text { branches per plant }\end{array}$ \\
\hline \multirow{2}{*}{$\begin{array}{l}\text { Range } \\
\text { value }\end{array}$} & Parents & 46.00 to 55.33 & 53.00 to 71.33 & 56.67 to 76.00 & 74.00 to 82.67 & 7.07 to 9.33 \\
\hline & Hybrids & 43.67 to 68.00 & 50.67 to 74.67 & 54.67 to 79.33 & 70.00 to 97.00 & 8.20 to 13.33 \\
\hline \multirow{2}{*}{$\begin{array}{l}\text { Mean } \\
\text { value }\end{array}$} & Parents & 50.46 & 58.04 & 62.67 & 78.33 & 8.18 \\
\hline & Hybrids & 50.46 & 57.48 & 61.93 & 78.70 & 9.58 \\
\hline \multirow{3}{*}{\multicolumn{2}{|c|}{$\begin{array}{l}\text { Top three } F_{1} \text { parents } \\
\text { with their mean value }\end{array}$}} & DVRT-1 (46.00) & DVRT-1 (53.00) & DVRT-1 (56.67) & DVRT-1 (74.00) & CHFT-60 (9.33) \\
\hline & & DVRT-2 (46.33) & DVRT-2 (53.33) & DVRT-2 (58.00) & DVRT-2 (76.00) & DVRT-2 (8.80) \\
\hline & & CHFT-71 (49.67) & CHFT-71 (56.00) & CHFT-71 (61.00) & CHFT-79 (76.00) & CHFT-77 (8.67) \\
\hline \multirow{5}{*}{\multicolumn{2}{|c|}{$\begin{array}{c}\text { Top five } F_{1} \text { hybrids } \\
\text { with their mean value }\end{array}$}} & $\begin{array}{c}\text { DVRT-1 x CHFT-50 } \\
(43.67)\end{array}$ & $\begin{array}{c}\text { DVRT-1 x CHFT-50 } \\
(50.67)\end{array}$ & $\begin{array}{c}\text { DVRT-1 x CHFT-50 } \\
(54.67)\end{array}$ & $\begin{array}{c}\text { DVRT-1 x CHFT-50 } \\
(70.00)\end{array}$ & $\begin{array}{c}\text { DVRT-2 x CHFT-60 } \\
(13.33)\end{array}$ \\
\hline & & $\begin{array}{c}\text { DVRT-1 x CHFT-60 } \\
(44.67)\end{array}$ & $\begin{array}{c}\text { CHFT-77 x H-86 } \\
(51.33)\end{array}$ & $\begin{array}{c}\text { CHFT-77 x H-86 } \\
(55.33)\end{array}$ & $\begin{array}{c}\text { CHFT-77 x CHFT-71 } \\
(71.67)\end{array}$ & $\begin{array}{c}\text { DVRT-2 x CHFT-77 } \\
(12.27)\end{array}$ \\
\hline & & $\begin{array}{c}\text { CHFT-77 x CHFT-71 } \\
(45.67)\end{array}$ & $\begin{array}{c}\text { CHFT-60 x CHFT-71 } \\
(52.33)\end{array}$ & $\begin{array}{c}\text { CHFT-60 x CHFT-71 } \\
(56.67)\end{array}$ & $\begin{array}{c}\text { CHFT-77 x H-86 } \\
(72.67)\end{array}$ & $\begin{array}{c}\text { CHFT-77 x CHFT-60 } \\
(12.20)\end{array}$ \\
\hline & & $\begin{array}{c}\text { CHFT-60 } \times \text { CHFT-71 } \\
(46.00\end{array}$ & $\begin{array}{c}\text { CHFT- } 77 \times \text { CHFT-79 } \\
(53.33 \text { days })\end{array}$ & $\begin{array}{c}\text { CHFT }-77 \times \text { CHFT-79 } \\
(58.00 \text { days })\end{array}$ & $\begin{array}{c}\text { CHFT-60 } \times \text { CHFT-79 } \\
(74.67 \text { days })\end{array}$ & $\begin{array}{c}\text { DVRT- } 2 \times \text { CHFT-79 } \\
(11.73)\end{array}$ \\
\hline & & $\begin{array}{c}\text { CHFT-77 } \times \text { CHFT-79 } \\
(47.33\end{array}$ & $\begin{array}{c}\text { CHFT-77 } \times \text { CHFT-71 } \\
(53.67 \text { days }) .\end{array}$ & $\begin{array}{c}\text { DVRT- } 1 \times \text { CHFT-77 } \\
\text { (58.33 days }) .\end{array}$ & $\begin{array}{c}\text { CHFT-60 } \times \text { CHFT-71 } \\
(74.67 \text { days })\end{array}$ & $\begin{array}{c}\text { H- } 86 \times \text { CHFT }-50 \\
(10.87)\end{array}$ \\
\hline \multicolumn{2}{|c|}{ C.V. (\%) } & 10.16 & 8.96 & 8.04 & 7.01 & 11.37 \\
\hline \multicolumn{2}{|c|}{ S.E.m \pm} & 2.99 & 2.98 & 2.88 & 3.19 & 0.61 \\
\hline
\end{tabular}


Table.3 Range, mean values, top 3 parents and 5 best hybrids for yield and its attributing traits

\begin{tabular}{|c|c|c|c|c|c|c|}
\hline \multicolumn{2}{|c|}{ Particulars } & $\begin{array}{c}\text { Number of locules } \\
\text { per fruit }\end{array}$ & Pericarp thickness & Plant height & Fruit length & Fruit girth \\
\hline \multirow{2}{*}{$\begin{array}{l}\text { Range } \\
\text { value }\end{array}$} & Parents & 2.33 to 5.13 & 0.43 to 0.51 & 46.83 to 87.73 & 3.70 to 5.16 & 4.81 to 6.11 \\
\hline & Hybrids & 2.93 to 6.33 & 0.42 to 0.70 & 64.97 to 119.87 & 3.90 to 5.55 & 4.89 to 8.03 \\
\hline \multirow{2}{*}{$\begin{array}{l}\text { Mean } \\
\text { value }\end{array}$} & Parents & 3.83 & 0.46 & 67.24 & 4.44 & 5.48 \\
\hline & Hybrids & 3.85 & 0.55 & 89.28 & 4.50 & 5.63 \\
\hline \multirow{3}{*}{\multicolumn{2}{|c|}{$\begin{array}{c}\text { Top three parents with } \\
\text { their mean value }\end{array}$}} & CHFT-77 (5.13) & CHFT-79 (0.51) & CHFT-60 (87.73) & CHFT-50 (5.16) & $\mathrm{H}-86(6.11)$ \\
\hline & & DVRT-2 (4.40) & H-86 (0.49) & CHFT-71 (76.33) & H-86 (5.07) & CHFT-77 (5.91) \\
\hline & & DVRT-1 (4.20) & CHFT-71 (0.47) & CHFT-79 (76.07) & CHFT-79 (4.67) & DVRT-2 (5.83) \\
\hline \multirow{5}{*}{\multicolumn{2}{|c|}{$\begin{array}{c}\text { Top five } F_{1} \text { hybrids } \\
\text { with their mean value }\end{array}$}} & $\begin{array}{c}\text { DVRT-2 x CHFT-77 } \\
(6.33)\end{array}$ & $\begin{array}{c}\text { DVRT-2 x H-86 } \\
(0.70)\end{array}$ & $\begin{array}{c}\text { CHFT-60 x CHFT-50 } \\
(119.87)\end{array}$ & $\begin{array}{c}\text { DVRT-2 x CHFT-77 } \\
(5.55)\end{array}$ & $\begin{array}{c}\text { DVRT-2 x CHFT-77 } \\
(8.03)\end{array}$ \\
\hline & & $\begin{array}{c}\text { DVRT-1 x CHFT-77 } \\
(4.73)\end{array}$ & $\begin{array}{c}\text { DVRT-2 x CHFT-77 } \\
(0.68)\end{array}$ & $\begin{array}{c}\text { CHFT-60 x CHFT-71 } \\
(117.45)\end{array}$ & $\begin{array}{c}\text { DVRT-2 x H-86 } \\
(5.33)\end{array}$ & $\begin{array}{c}\text { DVRT-2 x H-86 } \\
(6.61)\end{array}$ \\
\hline & & $\begin{array}{c}\text { CHFT-79 x H-86 } \\
(4.53)\end{array}$ & $\begin{array}{c}\text { CHFT-79 x CHFT-71 } \\
(0.67)\end{array}$ & $\begin{array}{c}\text { DVRT-2 x CHFT-79 } \\
(112.20)\end{array}$ & $\begin{array}{c}\text { CHFT-79 x H-86 } \\
(5.29)\end{array}$ & $\begin{array}{c}\text { CHFT-79 x H-86 } \\
(6.32)\end{array}$ \\
\hline & & $\begin{array}{c}\text { DVRT- } 1 \times \mathrm{H}-86 \\
(4.27)\end{array}$ & $\begin{array}{c}\text { CHFT-77 x CHFT-79 } \\
(0.63 \mathrm{~cm})\end{array}$ & $\begin{array}{c}\text { DVRT-2 } \times \text { CHFT-60 } \\
(108.93 \mathrm{~cm})\end{array}$ & $\begin{array}{c}\text { DVRT }-2 \times \text { CHFT }-71 \\
(4.89 \mathrm{~cm})\end{array}$ & $\begin{array}{c}\text { CHFT-77 } \times \text { CHFT-79 } \\
(6.04)\end{array}$ \\
\hline & & $\begin{array}{c}\text { DVRT }-1 \times \text { CHFT-50 } \\
(4.20)\end{array}$ & $\begin{array}{c}\text { CHFT-60 } \times \text { CHFT-71 } \\
(0.61)\end{array}$ & $\begin{array}{c}\text { H-86 } \times \text { CHFT-50 } \\
(108.12)\end{array}$ & $\begin{array}{c}\text { CHFT-79 } \times \text { CHFT-71 } \\
(4.84)\end{array}$ & $\begin{array}{c}\text { DVRT- } \times \text { CHFT-71 } \\
(5.92)\end{array}$ \\
\hline \multicolumn{2}{|c|}{ C.V. (\%) } & 10.23 & 9.43 & 10.53 & 10.46 & 9.28 \\
\hline \multicolumn{2}{|c|}{ S.E.m \pm} & 0.23 & 0.03 & 5.13 & 0.27 & 0.30 \\
\hline
\end{tabular}


Table.4 Range, mean values, top 3 parents and 5 best hybrids for yield and its attributing traits

\begin{tabular}{|c|c|c|c|c|c|c|c|}
\hline \multicolumn{2}{|c|}{ Particulars } & \multirow{2}{*}{$\begin{array}{c}\text { Fruit weight } \\
51.08 \text { to } 105.37\end{array}$} & \multirow{2}{*}{$\begin{array}{c}\begin{array}{c}\text { Number of } \\
\text { fruits per plant }\end{array} \\
41.40 \text { to } 48.20\end{array}$} & \multirow{2}{*}{$\begin{array}{c}\begin{array}{c}\text { Fruit yield per } \\
\text { plant }\end{array} \\
1.58 \text { to } 3.94\end{array}$} & \multirow{2}{*}{$\begin{array}{c}\begin{array}{c}\text { Total soluble } \\
\text { solids }(\%)\end{array} \\
3.44 \text { to } 3.99 \\
\end{array}$} & \multirow{2}{*}{$\begin{array}{c}\begin{array}{c}\text { Lycopene } \\
\text { content } \\
\text { (mg/100g) }\end{array} \\
4.28 \text { to } 11.40\end{array}$} & \multirow{2}{*}{$\begin{array}{c}\begin{array}{c}\boldsymbol{\beta} \text {-carotene } \\
\text { content } \\
\text { (mg/100g) }\end{array} \\
0.91 \text { to } 3.25\end{array}$} \\
\hline Range & Parents & & & & & & \\
\hline value & Hybrids & 54.59 to 209.67 & 30.88 to 92.83 & 1.19 to 9.13 & 3.15 to 4.47 & 2.46 to 21.36 & 0.67 to 3.62 \\
\hline \multirow{2}{*}{$\begin{array}{c}\text { Mean } \\
\text { value }\end{array}$} & Parents & 83.98 & 44.73 & 2.92 & 3.69 & 7.64 & 2.20 \\
\hline & Hybrids & 89.42 & 55.84 & 4.04 & 3.70 & 8.47 & 1.72 \\
\hline \multirow{3}{*}{\multicolumn{2}{|c|}{$\begin{array}{c}\text { Top three } \\
\text { parents with } \\
\text { their mean value }\end{array}$}} & H-86 (105.37) & $\begin{array}{c}\text { CHFT-71 } \\
(48.20)\end{array}$ & H-86 (3.94) & $\begin{array}{c}\text { CHFT-60 } \\
(3.99)\end{array}$ & CHFT-79 (11.40) & H-86 (3.25) \\
\hline & & $\begin{array}{c}\text { CHFT-77 } \\
(99.67)\end{array}$ & $\begin{array}{c}\text { CHFT-60 } \\
(47.80)\end{array}$ & CHFT-71(3.71) & $\begin{array}{c}\text { CHFT-71 } \\
(3.81)\end{array}$ & H-86 (9.40) & CHFT-50 (2.91) \\
\hline & & $\begin{array}{c}\text { CHFT-71 } \\
(96.37) \\
\end{array}$ & DVRT-2 (45.53) & CHFT-77 (3.56) & $\begin{array}{c}\text { CHFT-79 } \\
(3.77) \\
\end{array}$ & CHFT-50 (9.12) & CHFT-71 (2.82) \\
\hline \multirow{5}{*}{\multicolumn{2}{|c|}{$\begin{array}{c}\text { Top five } F_{1} \\
\text { hybrids with } \\
\text { their mean value }\end{array}$}} & $\begin{array}{c}\text { DVRT-2 x } \\
\text { CHFT-77 } \\
(209.67) \\
\end{array}$ & $\begin{array}{c}\text { CHFT-60 x } \\
\text { CHFT-50 } \\
(92.83) \\
\end{array}$ & $\begin{array}{c}\text { DVRT-2 x } \\
\text { CHFT-77 (9.13) }\end{array}$ & $\begin{array}{c}\text { DVRT-2 x } \\
\text { CHFT-60 } \\
(4.47) \\
\end{array}$ & $\begin{array}{c}\text { CHFT-79 x } \\
\text { CHFT-50 (21.36) }\end{array}$ & $\begin{array}{c}\text { DVRT-2 x } \\
\text { CHFT-50 (3.62) }\end{array}$ \\
\hline & & $\begin{array}{l}\text { DVRT-2 x H- } \\
86(164.94)\end{array}$ & $\begin{array}{c}\text { CHFT-60 x } \\
\text { CHFT-71 } \\
(82.70)\end{array}$ & $\begin{array}{c}\text { DVRT- } 2 \times \mathrm{H}-86 \\
(6.86)\end{array}$ & $\begin{array}{c}\text { DVRT-1 x } \\
\text { CHFT-71 } \\
(4.39)\end{array}$ & $\begin{array}{c}\text { DVRT-1 x } \\
\text { CHFT-50 (15.13) }\end{array}$ & $\begin{array}{c}\text { CHFT-79 x } \\
\text { CHFT-50 (2.95) }\end{array}$ \\
\hline & & $\begin{array}{l}\text { CHFT-79 x H- } \\
86(124.39)\end{array}$ & $\begin{array}{l}\text { H-86 x CHFT- } \\
71(79.27)\end{array}$ & $\begin{array}{c}\text { CHFT-79 x H-86 } \\
(6.47)\end{array}$ & $\begin{array}{c}\text { CHFT-79 x } \\
\text { CHFT-50 } \\
(4.31)\end{array}$ & $\begin{array}{c}\text { DVRT-2 x } \\
\text { CHFT-71 (14.61) }\end{array}$ & $\begin{array}{c}\text { DVRT-1 x } \\
\text { CHFT-50 (2.92) }\end{array}$ \\
\hline & & $\begin{array}{c}\text { DVRT-2 } \times \\
\text { CHFT-71 } \\
(101.33) \\
\end{array}$ & $\begin{array}{c}\text { CHFT-79 } \times \\
\text { CHFT-50 } \\
(72.55)\end{array}$ & $\begin{array}{c}\text { CHFT-60 } \times \\
\text { CHFT-71 }(5.92)\end{array}$ & $\begin{array}{c}\text { DVRT-2 } \times \\
\text { CHFT-79 } \\
(4.07)\end{array}$ & $\begin{array}{c}\text { DVRT-1 } \times \\
\text { CHFT-60 }(13.50)\end{array}$ & $\begin{array}{c}\text { CHFT-71 } \times \\
\text { CHFT-50 }(2.80)\end{array}$ \\
\hline & & $\begin{array}{c}\text { DVRT-2 } \times \\
\text { CHFT-79 } \\
(101.14) \\
\end{array}$ & $\begin{array}{l}\text { H- } 86 \times \text { CHFT- } \\
\quad 50(72.27)\end{array}$ & $\begin{array}{c}\mathrm{H}-86 \times \text { CHFT-71 } \\
(5.80)\end{array}$ & $\begin{array}{c}\text { H-86 } \times \text { CHFT }- \\
71(3.97)\end{array}$ & $\begin{array}{c}\text { DVRT }-2 \times \mathrm{H}-86 \\
(10.82)\end{array}$ & $\begin{array}{l}\text { H-86 x CHFT- } \\
50(2.70)\end{array}$ \\
\hline \multicolumn{2}{|c|}{ C.V. $(\%)$} & 13.87 & 14.47 & 14.57 & 5.15 & 4.52 & 4.91 \\
\hline \multicolumn{2}{|c|}{ S.E.m \pm} & 7.06 & 4.46 & 0.32 & 0.11 & 0.22 & 0.05 \\
\hline
\end{tabular}


Results are in agreement with Singh et al., (2014) and Lekshmi and Celine (2015). The highest number of fruit per plant observed in CHFT-60 x CHFT-50 (92.83), which was statistically at par with CHFT-60 $\times$ CHFT71(82.70). The fruit yield per plant ranged from 1.58 to $3.94 \mathrm{~kg}$ for parents and 1.19 to $9.13 \mathrm{~kg}$ in $\mathrm{F}_{1}$ hybrids. Among the parental lines, the highest fruit yield per plant was recorded in $\mathrm{H}-86$ (3.94 kg). The best hybrid regarding fruit yield performance was DVRT2 x CHFT-77 $(9.13 \mathrm{~kg})$, followed by DVRT-2 x H-86 (6.86 kg) and CHFT-79 x H-86 (6.47 $\mathrm{kg}$ ). Results of increased number of fruits per plant and yield are in concurrence with the findings of Farooq et al., (2012); Shankar et al., (2013); Islaam et al., (2014) and Dagade, et al., (2015).

Among the parental lines, highest total soluble solid was recorded for CHFT-60 (3.99 $\%)$. Out of $\mathrm{F}_{1}$ hybrids, highest total soluble solid was observed for DVRT-2 x CHFT-60 (4.47\%), which was statistically at par with DVRT-1 $\times$ CHFT-71 (4.39 \%) and CHFT-79 $\times$ CHFT-50 (4.31\%). Chattopadhyay et al., (2013) and Chernet et al., (2014) also reported the similar findings. The lycopene content among the parents ranged from 4.28 to $11.40 \mathrm{mg} / 100 \mathrm{~g}$ and among hybrids, it was between 2.46 to $21.36 \mathrm{mg} / 100 \mathrm{~g}$. Out of 28 hybrids, highest lycopene content was recorded for CHFT-70 x CHFT-50 (21.36 $\mathrm{mg} / 100 \mathrm{~g}$ ) followed by DVRT-1 x CHFT-50 (15.13 mg/100g) and DVRT-2 x CHFT-71 $(14.61 \mathrm{mg} / 100 \mathrm{~g})$. The findings are in accordance with Panthee et al., (2015). The $\beta$ carotene content ranged from 0.91 to 3.25 $\mathrm{mg} / 100 \mathrm{~g}$ and 0.67 to $3.62 \mathrm{mg} / 100 \mathrm{~g}$ in parents and $F_{1}$ hybrids, respectively. The highest $\beta$ carotene content found in hybrids DVRT-2 $\mathrm{x}$ CHFT-50 (3.62 mg/100g) followed by CHFT79 x CHFT-50 (2.95 mg/100g) and DVRT-1 x CHFT-50 (2.92 mg/100g). Similar findings were also made by Jindal et al., (2015) and Lekshmi and Celine (2015).
High estimates of heritability and genetic advance as per cent over mean were noticed for most of the traits indicates that their inheritance and phenotypic selection for their improvement could be achieved by simple breeding methods. From the above findings, it is concluded that parents DVRT-1 and CHFT60 for earliness; DVRT-2 and H-86 for fruits yield; CHFT-50 for lycopene and $\beta$-carotene content, were found superior among all the parents. These could be used in as one of the parent to produce high yielding and better quality hybrids, as well as in varietal improvement programmes. Hybrid DVRT-2 x CHFT-77, showed best for high yield and CHFT-60 x CHFT-71 for high yield and earliness. Average yield, earliness and quality traits observed in hybrid H-86 x CHFT-50. CHFT-79 x CHFT-50 showed high yield, TSS and lycopene content. For high lycopene and $\beta$-carotene, DVRT-1 x CHFT-50 found superior. These crosses may be exploited as commercial hybrids, after testing with few more in multilocation trial to confirm their potentiality stability over different agroclimatic situations. Therefore, it is suggested that hybrid breeding approaches might be more rewarding because hybrids advocate increased yield and other related traits in comparison with parents.

\section{Acknowledgement}

The authors wish to express their gratitude to IIVR, Varanasi, U.P. (India) and CHF, CAU, Pasighat, A.P. (India) for providing germplasm for conducting present experiment, as well as the Department of Vegetable Science, College of Horticulture and Forestry, CAU, Pasighat, A.P. (India) for providing facility to conduct this experiment.

\section{References}

Ahmad, M., Khan, B. A., Iqbal, M., Khan Z. U., Kanwal A., Saleem M. and 
Khurshid I. 2016. Study of Genetic Variability, Heritability and Genetic Advance in $F_{1}$ Generation of Tomato. Food Science and Quality Management. 47, 22-25.

Allard, R. W., 1960. Principles of plant breeding. J. Wiley and Sons, New York. Anonymous, 2015. http://nhb.gov.in/PDFViwer.aspx?enc= 3ZOO8K5CzcdC/Yq6HcdIxC0U1kZZe nFuNVXacDLxz28.

Burton, G. W., 1952. Quantitative inheritance in grasses. Proceeding on 6th International Grass Conservation, pp. $277-283$.

Chattopadhyay, A., Chakraborty, I., Siddique, W. 2013. Characterization of Determinate Tomato Hybrids: Search for Better Processing Qualities. Journal of Food Processing Technology. 4, 222.

Chernet, S., Belew, D. and Abay, F. 2014. Performance Evaluation and Path Analysis Studies in Tomato (Solanum lycopersicon L.) Genotpes under Humera, Northern Ethiopia Condition. World Journal of Agricultural Research. 2(6):267-271.

Dagade, S.B., Barad, A.V., Dhaduk, L.K. and Hariprasanna, K. 2015. Estimates of hybrid vigour and inbreeding depression for fruit nutritional characters in tomato. International Journal of Science, Environment and Technology, 4(1): 114-124.

Farooq, A. M., Nasir, I. A., Tabassum, B., Tariq, M., Qamar, Z., Khan, M. A., Nadeem, A., Haider, M.S., Anwar, W., Javed, M.A. and Husnain, T. 2012. Development and comparative studies of double cross tomato hybrids. African Journal of Agricultural Research. 7(37): 5259-5264.

Gomez, K.A., and Gomez, A.A. 1983. Statistical procedure for agricultural research 2nd ed., John Wiley and Sons, New York. Pp. 375-427.
Islam, M.S., Hasanuzzaman, M., Rahman, M.S., Shoma1, J.F. and Ali, L. 2014. Performance of different productive hybrids in tomato (Lycopersicon esculentum). Eco-friendly Agriculture Journal. 7(11): 151-153.

Jindal, S. K., Dhaliwal, M.S. and Chawla, N. 2015. Comparative Performance of Different Tomato Hybrids under Naturally Ventilated Polyhouse. International Journal of Horticulture. 5(14): 112.

Johnson, H.W., Robinson, H.F. and Comstock, R.E. 1955. Estimates of genetic and environmental variability in soybean. Agronomy Journal. 47(7): 314-318.

Lekshmi, S. L., and Celine, V. A. 2015. Evaluation of tomato hybrids for fruit, yield and quality traits under polyhouse conditions. International Journal of Applied and Pure Science and Agriculture. 1(7): 58-64.

Meena, O. P., and Bahadur, V. 2014. Assessment of genetic variability, heritability and Genetic advance among tomato (Solanum lycopersicum L.) germplasm. The bioscan. 9(4): 16191623 ,

Pandey, V.C., Ram C.N., Deo, C., Chakravarti, S.K. And Rao, J. K. 2016. Screening of germplasm for quality and yield traits in tomato (Solanum lycopersicum L.). The Bioscan, 11(2): 925-928.

Panthee, D., Perkins-Veazie, P., Anderson, C. and Ibrahem, R. 2015. Diallel analysis for lycopene content in the different colored parents in tomato. American Journal of Plant Sciences, 6, 1483-1492.

Pedapati, A., Reddy, R.V.S.K., Babu, J.D., Kumar, S.S. and Sunil, N. 2013. Combining ability analysis for yield and physiological drought related traits in tomato (Solanum lycopersicum L.) under moistures stress. The Bioscan, 
8(4): 1537-1544.

Prajapati, S., Tiwari, A., Kadwey, S. and Jamkar, T. 2015. Genetic Variability, Heritability and Genetic Advance in Tomato (Solanum Lycopersicon Mill.). International Journal of Agriculture, Environment and Biotechnology. 8(2): 245-251.

Rao, E.S., Munshi, A.D., Singh, B. and Kumar, R. 2007. Studies on heterosis and combining ability for yield and resistance to early blight in tomato. Indian Journal of Horticulture, 64(3): 331-334.

Shankar, A., Reddy, R.V.S.K., Sujatha, M., Pratap, M. 2013. Combining ability and gene action studies for yield and yield contributing traits in tomato (Solanum lycopersicum L.). Helix. 6, 431 - 435.

Singh, T., Singh, N., Bahuguna, A., Nautiyal,
M. and Sharma, V.K. 2014. Performance of tomato (Solanum lycopersicon L.) hybrids for growth, yield and quality inside polyhouse under mid hill condition of Uttarakhand. American Journal of Drug Discovery and Development. Pp 1-8.

Swaminathan, M.S., and Bhavani, R.V. 2013. Food production \& availability Essential prerequisites for sustainable food security. Indian Journal of Medical Research. 138(3): 383-391.

Yadav, S. K., Singh, B. K., Baranwal, D. K. and Solankey, S. S. 2013. Genetic study of heterosis for yield and quality components in tomato (Solanum lycopersicum). African Journal of Agricultural Research. 8(44): 55855591.

\section{How to cite this article:}

Sanket Kumar, Vikas Singh, Praveen Kumar Maurya, B. Ashok Kumar and Yadav, P.K. 2017. Evaluation of F1 Hybrids along with Parents for Yield and Related Characteristics in Tomato (Solanum lycopersicum Child). Int.J.Curr.Microbiol.App.Sci. 6(9): 2836-2845. doi: https://doi.org/10.20546/ijcmas.2017.609.348 